\title{
A real time phonetically based spoken word recognition system and recognizer for unspecified speakers
}

\author{
Sei-ichi Nakagawa* and Toshiyuki Sakai** \\ *School of Information Engineering, Toyohashi University of Technology, \\ Toyohashi, 440 Japan \\ **Department of Information Science, Kyoto University, \\ Kyoto, 606 Japan
}

(Received 1 March 1981)

\begin{abstract}
We have developed a real time phonetically based spoken word recognition system on a mini-computer and a recognition equipment which consisted of a filter bank and a microcomputer. If the vocabulary size of the word recognition system is enlarged to about several hundreds, not only the recognition time becomes very long by increasing an amount of processing but also the correct rate of recognition decreases. To cope with these weak points, we adopted the method which reduced candidate words in the vocabulary by means of pre-matching using both local and global features of a spoken word. Our system is based on the phoneme classification, that is, hierarchical linguistic knowledge. To extend this approach to connected words recognition, we developed a word boundary detection method by using the pitch and energy contours. In an experiment on the recognition using 100 Japanese city names or ten digits, the system recognized the names (digits) correctly at the rate of $84 \%(98 \%)$ for unspecific speakers and recognized two connected digits at the rate of $83 \%$ in real time. Furthermore, we constructed a special equipment of speech recognition which was based on the system. In experiments on word recognition of 32 city names, the recognizer recognized them correctly at the rate of $93.4 \sim 94.4 \%$ for unspecific male and female speakers.
\end{abstract}

PACS number: 43. 70. Sc

\section{INTRODUCTION}

In automatic word recognition, if the word input pattern is regarded as a point in the pattern space, a word to word matching is adopted. Therefore, for limited speakers, the recognizer can avoid the problem of coarticulation, and it is fairly easy to recognize spoken words in a limited vocabulary. Recent researches in word recognition have focussed on the following goals:

(1) enlargement of the vocabulary size.

(2) reduction of the amount of computation and storage.

(3) development for a general word recognition scheme on the basis of phenome recognition.

(4) normalization or learning of speaker differ- ences for unspecified speakers.

(5) extension to recognition of connected words.

(6) construction of a recognizer with microcomputer or LSI circuits.

In addition to these points, effective usages of the word dictionary and fast matching algorithms have also been studied as a part of the research. For matching an input pattern with a reference pattern, we believe that a matching method using DP (dynamic programming) is one of the best algorithms suited for automatic word recognition. We also believe this because all DP matching algorithms preserve the properties of speech sounds such as continuity and monotony in time, and they allow for nonlinear warping on the matching between two strings with different length. ${ }^{15)}$ The word to word matching method has the high performance for 
limited speakers. For unspecified speakers, however, its performance is comparable with a phonetically based word recognition method. A phonetically based method has some advantages such as small computation and storage, few learning samples for speaker adaptation and easier change of vocabulary.

We tried to consider all the subjects of (1) through (6) mentioned above. We described about the learning procedure for (4) in two other papers separately. ${ }^{1,2)}$ In this paper, we describe a phonetically based word recognition method for (3), a pre-matching method for (1) and (2), a word boundary detection method for (5) and a special equipment for (6). Although several methods have been tried for the speed-up of recognition, ${ }^{3,4)}$ they all were huristic methods. Our method uses both the local and global features of a spoken word.

Recently, several methods were proposed for the recognition of connected spoken words. ${ }^{5-8)}$ Almost all methods are based on pattern matching.
Rabiner et al. proposed the word boundary detection method of connected digits. However, this algorithm depended on the contents of vocabulary, that is, it was a digit-oriented algorithm. We propose a new word boundary detection method, applicable for any word sequence.

\section{SYSTEM OVERVIEW}

Figure 1 shows a block diagram of a real-time spoken word recognition system, which is composed of (a) the preliminary learning stage of the speaker differences and (b) the stage of spoken word recognition in real time. The latter stage consists of a phoneme classification component, a word recognition component from a phoneme string to a word, and a non-supervised learning part.

First, a new user of the system is requested to utter clearly Japanese five vowels $(/ \mathrm{a} /, / \mathrm{i} /, / \mathrm{u} /, / \mathrm{e} /$, $/ \mathrm{o} /$ ) and the syllabic nasal $/ \mathrm{N} /$ for the preliminary learning of his own voice. Since the inter-speaker

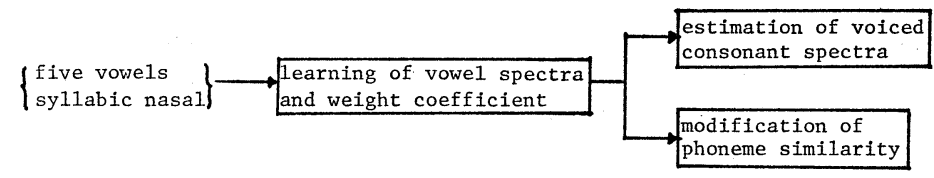

(a) learning stage (preliminary learning of speaker differences)

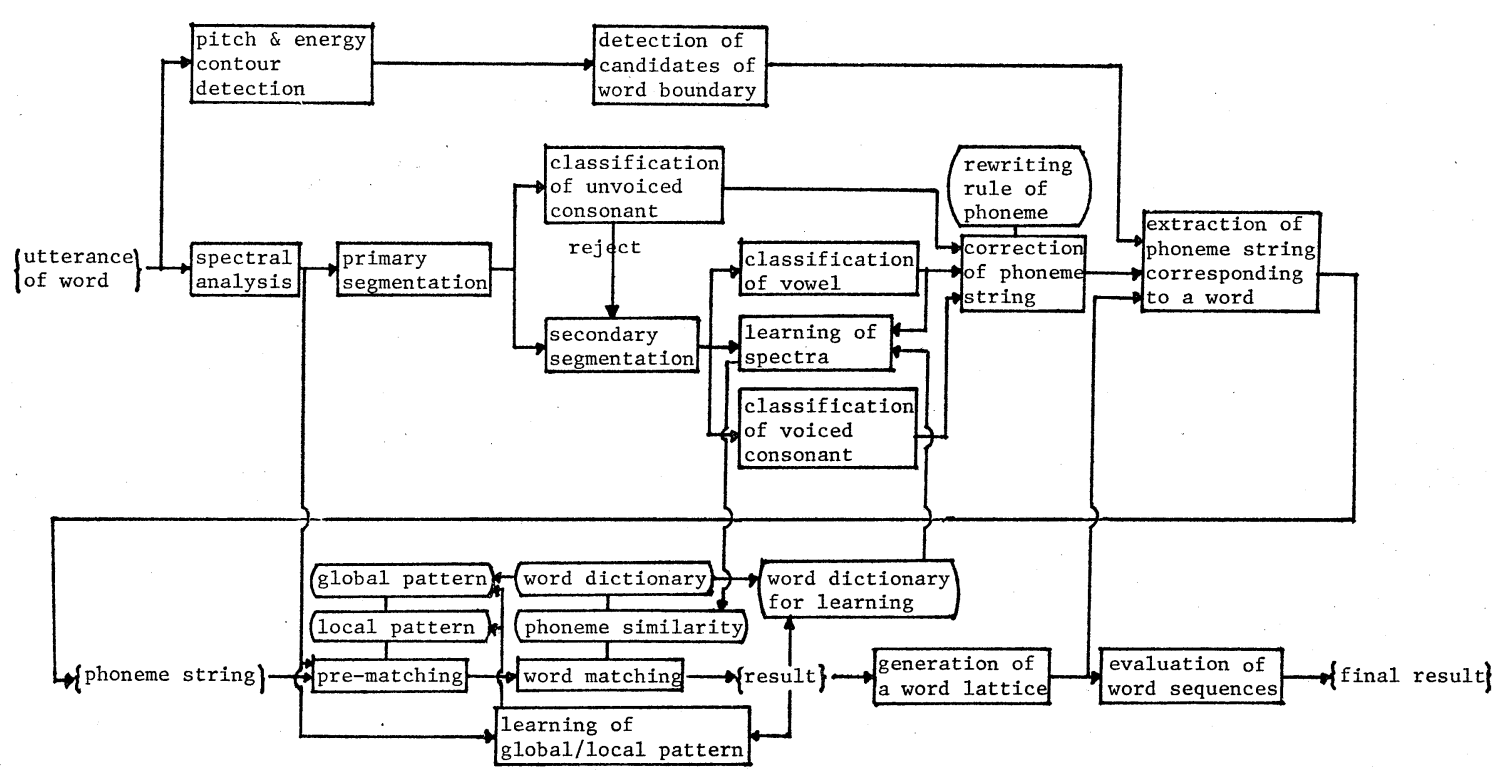

(b) recognition stage (with non-supervised learning)

Fig. 1 A real time phonetically based connected spoken word recognition system.

(a) Learning stage (preliminary learning of speaker differences), (b) recognition stage (with non-supervised learning). 


\section{S. NAKAGAWA and T. SAKAI: A SPOKEN WORD RECOGNITION SYSTEM AND RECOGNIZER}

spectral variation of the syllabic nasal is very large, ${ }^{97}$ it is learned as well as vowels. Note that it is uttered as $/ \mathrm{uN} /$, because we cannot utter it in isolation. The learned spectrum of the syllabic nasal is used for the estimation of voiced consonant spectra.

Second, after the learning process, he can utter any word in a vocabulary. In order to recognize in real time, the system employs a simple algorithm in the part of the phoneme classification component. The output of phoneme classification component is a string of segments, each consisting of 4 tuples: the first candidate of phonemes, the second candidate, degree of confidence of the first candidate and segment duration. Finally, the classified phoneme string is passed to the stage of word recognition.

The process for shortening of recognition time is carried out between the phoneme classification process and the word matching process. Before the word matching, an uttered word is examined about measured values of both local and global features of a spoken word to eliminate the most unlikely group of candidates from the vocabulary list (we call this pre-matching). Besides benefits of the shortening of recognition time, the correct rate of recognition would be improved by adding this pre-matching result to a final word matching result. The spectral patterns at the head and tail positions of spoken words were employed for the local features. The features such as the number of phonemes, the presence/absence of each vowel and consonant in the classified phoneme string, and the contour of energy envelope were employed for the global features.

The classified segment (phoneme) string is matched with an element string in a lexical entry by using the similarity between a segment and an element in the entry (we call this word matching). The best matching of all possible associations, which is calculated by a dynamic programming technique, is regarded as the likelihood of that word. The word to which the input has the largest likelihood among all possible words is regarded as the input word.

In the case of connected words, the system detects the word boundaries by using the pitch and energy contours, before the pre-matching and the word matching. The system makes up the phoneme string which corresponds to an isolated word from the detected word boundaries and the classified phoneme string. The extracted phoneme string is matched with all lexical entries. Since an over- lapped word lattice is obtained by this procedure, the system selects the best non-overlapping word sequence and regards it as the input.

The spectral patterns of vowels, weighting coefficients for linear discriminant functions and binary patterns for the pre-matching are learned for each speaker by using a non-supervised learning procedure in parallel with the recognition stage. And also, the phoneme similarity matrix and spectral patterns of voiced consonants are modified for each speaker by using the learned spectral patterns of vowels.

This system was implemented on a mini-computer (MELCOM-70, cycle time $=0.8 \mu \mathrm{s}$, core memory $=$ $32 \mathrm{~kW}$, cartridge disk $\times 2=5 \mathrm{MW}$ ). The algorithms were programmed with an assembly language. This program occupied about $10 \mathrm{~kW}$ and the work area about $10 \mathrm{~kW}$. The matching time required between a segment string and an element string in a lexical entry was about $50 \mathrm{~ms}$. This system can recognize 100 Japanese city names correctly at the rate of about $93 \%$ by using a non-supervised learning procedure in real time. ${ }^{2)}$

\section{SEGMENTATION AND PHONEME CLASSIFICATION}

Speech signals are first passed into a pre-emphasis circuit with a slope of 6-dB per octave below $1600 \mathrm{~Hz}$, because of improving to signal-to-noise ratio at high frequencies, and then fed into the 20-channel $1 / 4$ octave filter-bank. After they are full-waverectified and smoothed by the low-pass filter (cut-off frequency $=40 \mathrm{~Hz}$ ), the output waves are sampled at every $10 \mathrm{~ms}$ interval and digitized with an accuracy of 10 bits. The center frequencies of the 20 channels are from $210 \mathrm{~Hz}$ to $5660 \mathrm{~Hz}$.

The aim of phoneme classification is to segment input speech into a unit of phoneme (we call this unit a segment, that is, by a segment we mean a time portion of the utterance which is hypothesized to be a single phoneme), and assigns one of the phoneme categories to the unit. We classify Japanese phonemes into the following categories.

1. vowel $/ \mathrm{a} /, / \mathrm{i} /, / \mathrm{u} /, / \mathrm{e} /, / \mathrm{o} /$

2. semi-vowel $/ \mathrm{y} /, / \mathrm{w} /$

3. nasal $/ \mathrm{m} /, / \mathrm{n} /, / \mathrm{n} /$

4. voiced plosive $/ \mathrm{b} /, / \mathrm{d} /, / \mathrm{g} /$

5. liquid-like $/ \mathrm{r} /$

6. voiced fricative $/ z /, / \mathrm{d}_{z} /$

7. unvoiced plosive $/ \mathrm{p} /, / \mathrm{t} /, / \mathrm{k} /$ 

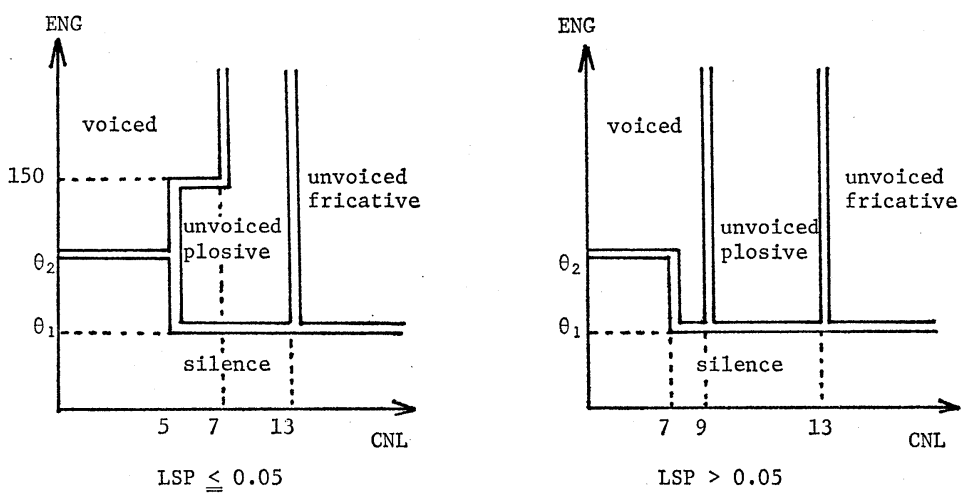

Fig. 2 Primary segmentation.

8. unvoiced fricative $/ \mathrm{s} /, / \mathrm{s} /$

9. affricate $/ \mathrm{ch} /, / \mathrm{ts} /$

10. aspirated $/ \mathrm{h} /$

11. syllabic nasal $/ \mathrm{N} /$

12. silence $/ . /, 1-1$

The silence $/ . /$ and $/-/$ corresponds to the closure of unvoiced plosive consonants and a choked sound, respectively. The system treats $|\mathrm{z}|$ and $\left|\mathrm{d}_{z} /,\right| \mathrm{s} \mid$ and $/ \mathrm{J} /$ or $/ \mathrm{ch} /$ and $/ \mathrm{ts} /$ as the same category $(/ \mathrm{z} /$, $/ \mathrm{s} /, / \mathrm{c} /)$. The system does not classify an unvoiced plosive group into a phoneme, therefore, we simply denote this group as $/ \mathrm{p} /$.

The primary segmentation is performed for analyzed speech signal. After the processing, the input (a sequence of short time spectra) is classified into one of silence, unvoiced plosive, unvoiced fricative and voiced sounds based on the energy and deviation around the low or high frequency of spectrum at every $10 \mathrm{~ms}$ (we call this spectral unit as a frame hereafter). Figure 2 illustrates the segmentation procedure. Where ENG, CNL, LSP, $\theta_{1}$, and $\theta_{2}$ are defined as follows. Let $x_{i}$ be the output value of the $i$-th channel at a frame.

$$
\begin{aligned}
\mathrm{ENG}= & \left(\sum_{i=1}^{20} x_{i}^{2}\right)^{1 / 2} \\
\mathrm{CNL}= & \text { the minimum channel number } j \text { which } \\
& \text { satisfied the next condition. } \\
& \sum_{i=1}^{j} x_{i} \geqq \mathrm{ENG} / 2 \\
\mathrm{LSP}= & \sum_{i=1}^{3} x_{i} / \mathrm{ENG} \\
\theta_{1}= & 10, \theta_{2}=15 \text { (at the beginning part of an } \\
& \text { utterance) } \\
\theta_{1}= & 20, \theta_{2}=30 \text { (at the ending part of an utter- }
\end{aligned}
$$

\section{ance)}

Since the pronounciation becomes ambiguous at the ending part of an utterance, the part is truncated a little early.

If a successive part of a sequence of classified phonemes is composed the same phonemes, they will be combined. On the other hand, if it is a irregular, it will be smoothed by using rewriting rules or phonological rules. The typical rules are Japanese simple rules of syllable consisting of successive phonemes such as $\mathrm{CV}, \mathrm{CyV}, \mathrm{VN}$ (C: consonant, $\mathrm{V}$ : vowel). The segment classified as an unvoiced group is further classified into one of the detailed group corresponding to each phoneme on the basis of the segment duration, the presence of silence in a preceding segment and spectral change, etc.

From segments classified into voiced groups, portions of voiced consonants are detected as one of the following; 1) long transient, 2) having weak energy with concave speech level. And the undetected portions for the consonants are regarded as vowels (secondary segmentation).

In the processing of vowels, the system computes a distance between each frame in voiced parts and each of six reference patterns: five vowels and the syllabic nasal (the syllabic nasal is treated like the five vowels in the following process). The cityblock distance (Chebyshev norm) is used as a distance measure. The calculation of this distance need not be multiplicative.

The nearest neighbors are selected as candidate phonemes and then ordered by application of the corresponding linear discriminant functions. The voiced consonant parts are classified on the basis of Euclidean distance. These phoneme classifica- 
tion procedure can be achieved for each frame within the sampling interval, $10 \mathrm{~ms}$.

A classified phoneme string in voiced parts is smoothed and merged by rewriting rules. An output of phoneme classification process is a sequence of segments, each consisting of 4 tuples: the first candidate of phonemes, the second candidate, degree of confidence of the first candidate and segment duration.

\section{PRE-MACHING}

Pre-matching should be a simple algorithm because this aims to reduce the recognition time. This procedure pre-selects candidate words for an uttered word from the vocabulary and passes only these words to the word matching procedure. We employed both local and global features of a spoken word for pre-matching. These features have not been used at the phoneme classification or word recognition, therefore, we can expect that prematching also brings the improvement of recognition performance by the following two reasons.

(1) Words which are similar to an uttered word on a classified segment string may be rejected by using local features.

(2) The correct rate of recognition is improved by adding these new measures of pre-matching to word matching score.

\subsection{Local Pattern of Spoken Word}

The spectral binary patterns of head and tail positions of a spoken word are employed for local features. In the head position, eight frames $(80 \mathrm{~ms})$ are divided into two intervals automatically at the most changing point on the basis of spectral changes. That is, the system computes the amount of spectral change between adjacent frames from the third frame to the sixth frame. Of course, spectral values of each frame are normalized in order to avoid the variation of speech power. The spectral patterns are averaged over all frames in each divided interval for every channel and then are transformed into a binary pattern at a certain threshold value. Thus, we obtain a spectral binary pattern of 40 bits. In almost case, the former part in the divided head parts corresponds to a consonant of $\mathrm{CV}$ (or $\mathrm{CyV}$ ) syllables and the latter part of a vowel. If the initial phoneme of a word is a vowel or a fricative, both former and latter parts are to be the same phoneme. However, we can expect that the latter part never contains the following CV syllables, because man requires the duration time of more than $80 \mathrm{~ms}$ to utter any CV syllable and vowel in the head position of Japanese word. Therefore, we can make these reference patterns from all CV syllables (about 100 syllables) of Japanese spoken in isolation.

In the tail position, the spectral patterns of the last six frames $(60 \mathrm{~ms})$ are averaged and transformed into a binary pattern in the same manner as the head position. We obtain a spectral binary pattern of 20 bits.

It has the advantage that these positions are exactly detected on any speed of an utterance and also these patterns are insensitive on contexts. Figure 3 illustrates an example of these positions and patterns.

\subsection{Global Pattern of Spoken Word}

\subsubsection{Number of phonemes in a spoken word}

Whatever the speed of an utterance is, the number of phonemes in a spoken word is constant. Therefore we can derive from the number of classified phonemes what kind of words was uttered. However we should note that the segmentation of our system yields errors.

\subsubsection{Kind of phonemes in a spoken word}

We can guess an uttered word by the kind of phonemes contained in a classified phoneme string. If the order of occurrence of phonemes is taken into consideration, the more exact guess would be possible, however, the algorithm to guess would become sophisticated by reason of the incompleteness of segmentation and classification. Therefore we transform the classified phoneme string into the following eight bit pattern (phonemic pattern).

vowel $(\mathrm{a}, \mathrm{i}, \mathrm{u}, \mathrm{e}, \mathrm{o})$......1 bit for each vowel. unvoiced consonant $(\mathrm{p}, \mathrm{t}, \mathrm{k}, \mathrm{s}, \mathrm{c}, \mathrm{h}) \ldots . .2 \mathrm{bit}$ voiced consonant ( $m, n, y, b, d, g, r, z) \ldots . .1$ bit If these phonemes appear as the first candidate phoneme of classified phoneme string, the corresponding bits are set 1 , if not, 0 (if two unvoiced consonants appear $\rightarrow 11$, one $\rightarrow 10$, zero $\rightarrow 00$ ). The reference pattern is translated from the word dictionary. Some examples are shown in Fig. 4.

\subsubsection{Contour of energy envelope}

The global contour of energy envelope of a spoken word is almost constant among speakers. Where the energy is defined as the root of square sum of 20-channel outputs. The length of a word is divided 


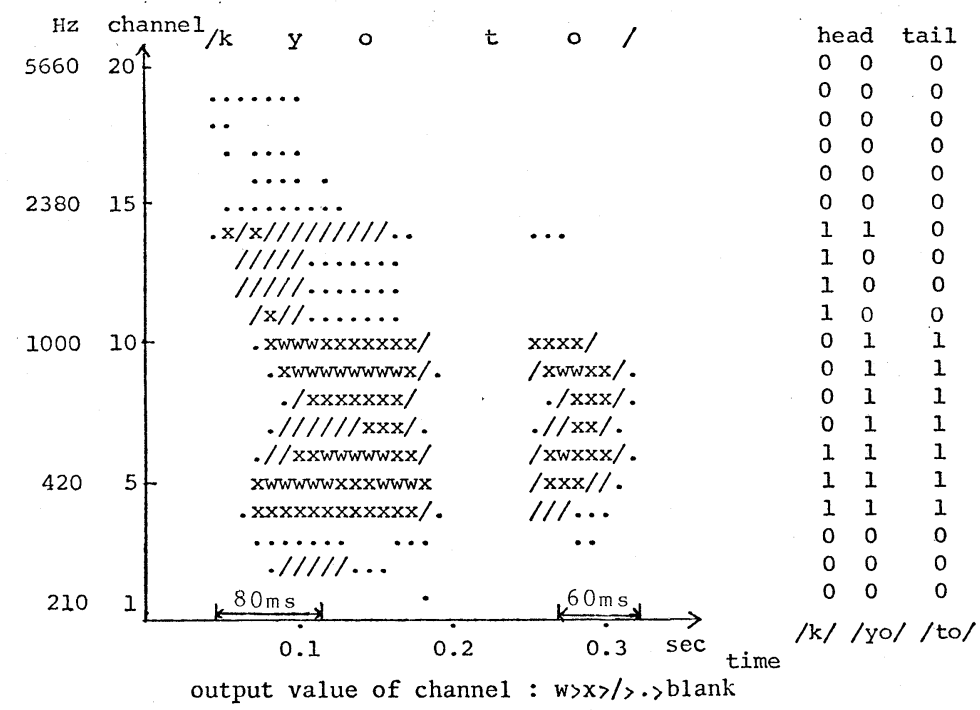

Fig. 3 Local spectral pattern.

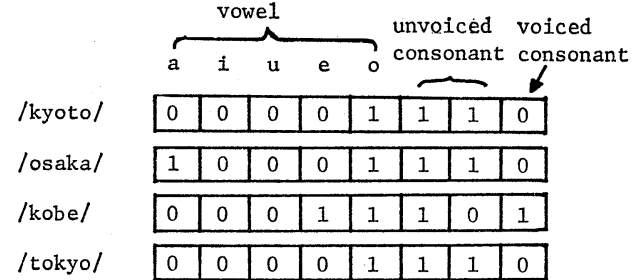

Fig. 4 Phonemic pattern.

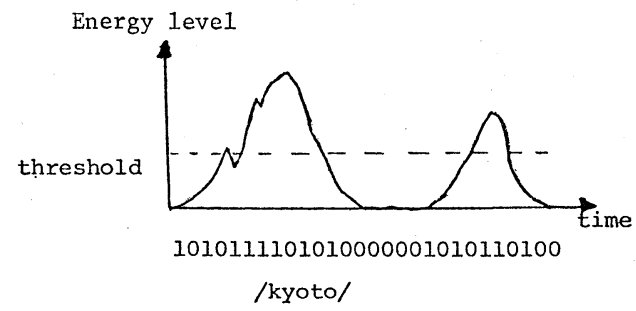

Fig. 5 Envelope pattern of speech energy. into 14 intervals linearly. The system checks for each interval is the slope of energy envelope is positive or negative and if the value of energy level is larger than the half of the largest value in all frames. For each interval, two bits are set according to the status mentioned above as follows: positive and larger $\rightarrow 11$, positive and smaller $\rightarrow 10$, negative and larger $\rightarrow 01$, and negative and smaller $\rightarrow 00$. Thus we obtain a binary pattern of 28 bits. Only the reference pattern of this feature cannot be generated automatically, that is, we must prepare the reference pattern for every word individually. Figure 5 illustrates an example of this pattern.

\subsection{Pre-Matching Procedure}

Pre-matching should be performed very quickly, because it aims to reduce the recognition time of a spoken word. Therefore we adopted the simple bit pattern and a simple algorithm. As described above, the features for pre-matching are represented by the number of phonemes in a spoken word and binary patterns of 96 bits ( 6 computer words); such as spectral patterns in the head and tail positions ( 40 bits and 20 bits), a phonemic pattern of 8 bits and a contour pattern of energy envelope of 28 bits. These binary patterns were compared in parallel with each reference pattern on the basis of Hamming distance. Let $\theta$ be the difference between the number of classified phonemes and the number of phonemes in the $n$-th lexical word. Let $\alpha, \beta, \gamma$ and $\delta$ be the Hamming distance between the binary patterns of a spoken word and the binary reference patterns of the $n$-th lexical word for above each feature respectively. The procedures consist of the following 6 steps.

(1) Check of the number of phonemes

If the number in the $n$-th lexical word is smaller than 9 and $\theta \geqq 4$, its lexical word is rejected. 


\section{S. NAKAGAWA and T. SAKAI: A SPOKEN WORD RECOGNITION SYSTEM AND RECOGNIZER}

If the number is greater than 10 and $\theta \geqq 5$, it is rejected.

(2) Check of the spectral binary pattern in the head position

If $\alpha \geqq 20$, its lexical word is rejected.

(3) Check of the spectral binary pattern in the tail position

If $\beta \geqq 12$, its lexical word is rejected.

(4) Check of the phonemic pattern

If $\gamma \geqq 5$, its lexical word is rejected.

(5) Check of the contour pattern of energy envelope If $\delta \geqq 12$, its lexical word is rejected.

(6) Check of the total bit patterns

If $\alpha+\beta+\gamma+\delta \geqq 36$, its lexical word is rejected.

\section{ISOLATED WORD RECOGNITION FROM PHONEME STRING}

\subsection{Similarity between Two Phonemes}

The phoneme classification is performed using statistics of the spectrum (means and covariance matrices in 20-dimensional vectors for making linear discriminant functions) for phonemes. Thus, if the phoneme classification part makes mistakes, we should consider that such errors are caused by the fact that the spectra obtained from an uttered phoneme are very similar to those of a misclassified phoneme. The errors are generally divided into three kinds: (a) substitution, (b) insertion, (c) omission. Word matching is fundamentally defined as the process which makes a one-to-one correspondence between each phoneme of a classified phoneme string and each phoneme of an entry in the word dictionary. To evaluate a degree of matching between two phonemes, we introduce a concept of similarity between two phonemes.

The Bhattacharyya distance is closely related to the confusion matrix constructed from the results of phoneme classification based on Bayes' rule. ${ }^{12}$ ) We calculate the similarity $S(i, j)$ for a pair of phoneme ( $i$ and $j \in$ voiced phonemes) by the linear transformation of the distance. If either $i$ or $j$ is unvoiced, $S(i, j)$ is derived from the confusion matrix. The resulting similarity matrix is shown in Table 1. The row "in" denotes phonemes in the lexicon, and the column "out," classified phonemes. In this table, the pseudo phoneme $/ * /$ is treated as an unvoiced plosive, except that it is not associated with the silence group.

\subsection{Word Dictionary}

Some phonemes in a word are often influenced by phoneme environments, while other phonemes are sometimes devocalized. By introducing the subphoneme ' $k$ ' in addition to the main-phoneme ' $I$ ', we denote these situations in the dictionary by $I / k(c)$. This notation means that the phoneme ' $I$ ' can be replaced by the phoneme ' $k$ ', where $c(0 \leqq c \leqq 1.0)$ means the weight or probability of the sub-phoneme ' $k$ '. Both phonemes are equally treated if it is 1.0 , and the sub-phoneme is neglected if it is 0 . By this description, we can represent an optional phoneme, i.e., one that is omissible or addible.

Table 2 shows some examples (ten digits) of the word dictionary. The special symbols $(+$ and - which will be explained in the next section) indicate changes of restrictions for word matching. These representations for given words are automatically constituted by the constructing rules of the word dictionary.

\subsection{Word Matching Procedure}

An output of the phoneme classification part is a sequence of segments, each of which consists of the first and second candidates of phonemes, the degree of confidence of the first candidate for the second candidate. The three constituents of the $j$-th segment in a string will be denoted by $J, l$ and $p$ $(0 \leqq p \leqq 1.0)$, respectively. An element by which a word in the word dictionary is described is either a main-phoneme or a sub-phoneme plus a weighting factor. The constituents of the $i$-th element of a lexical entry will be denoted by $I, k$ and $c$, respectively. In order to match a portion of a segment string against a word, we must first define the similarity between a segment and an element in the entry. This similarity is defined by the following equation:

$$
\begin{aligned}
& S(I, k, c ; J, l, p)= \\
& \max \left\{\begin{array}{l}
S(I, J) \\
c \cdot S(k, J) \\
p \cdot S(I, J)+(1-p) \cdot S(I, l) \\
p \cdot c \cdot S(k, J)+(1-p) \cdot c \cdot S(k, l)
\end{array}\right\}
\end{aligned}
$$

we simply denote $S(I, k, c ; J, l, p)$ as $S_{0}(i, j)$.

We make the following restrictions with respect to the matching between an input string and an element string in the word dictionary. These could be regarded as reasonable restrictions, judging from 


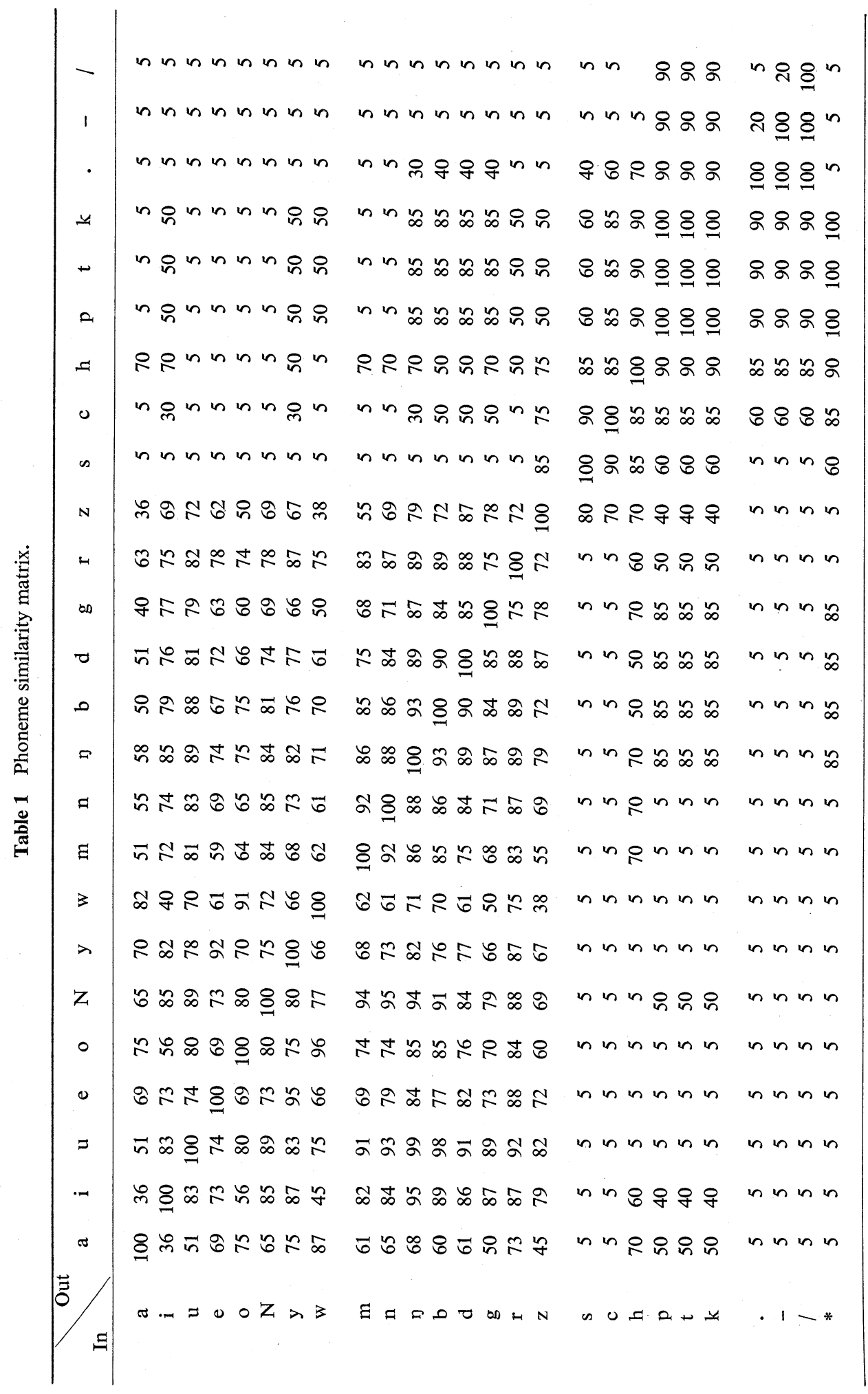


Table 2 Examples of entries in the Word Dictionary (10 digits).

\begin{tabular}{|c|c|c|}
\hline Word & $\begin{array}{c}\text { Sym- } \\
\text { bol }\end{array}$ & Phonemic representation \\
\hline ichi & 1 & i./c (1.0) $\mathrm{c} \mathrm{i}^{+-} / \mathrm{c}(1.0)$ \\
\hline ni & 2 & $\mathrm{n} \mathrm{i}$ \\
\hline san & 3 & $s$ a $N$ \\
\hline yon & 4 & $\mathrm{y} / \mathrm{g}(0.95)$ o $\mathrm{N}$. \\
\hline go & 5 & g o \\
\hline roku & 6 & $\mathrm{r} o . / \mathrm{k}(0.95) \mathrm{k} \mathrm{u}^{+-} / *(1.0)$ \\
\hline nana & 7 & $\mathrm{n} \mathrm{a} / \mathrm{N}(0.85) \mathrm{n} / \mathrm{a}(0.85) \mathrm{a}^{+} / \mathrm{N}(0.85)$ \\
\hline hachi & 8 & $\mathrm{~h} \mathrm{a} / \mathrm{N}(0.85) . / \mathrm{c}(1.0) \mathrm{c} \mathrm{i}^{+-} / \mathrm{c}(1.0)$ \\
\hline kyu & 9 & $\mathrm{k} / \mathrm{c}(0.95) \mathrm{y} / \mathrm{u}(0.95) \mathrm{u}$ \\
\hline rei & 0 & $\mathrm{r} / \mathrm{p}(0.85)$ e $\mathrm{i} / \mathrm{e}(0.95)$ \\
\hline
\end{tabular}

the performance of the phoneme classification.

(1) Except for elements marked with the symbol $(-)$, a vowel and the syllabic nasal in the word dictionary can be associated with three or less segments in a classified phoneme string.

(2) A consonant can be associated with two or less segments.

(3) Three or more successive elements cannot be associated with one segment except when there is an element marked with the symbol $(+)$.

Now, we consider how to calculate the likelihood for a spoken word in isolation. Let $L(i, j)$ be the highest cumulative similarity (or score), when considering the $i$-th element of the lexical entry for this word and the $j$-th segment of a classified phoneme string. In other words, $L(i, j)$ is determined by evaluating all possible paths from the grid point $(1,1)$ to the grid point $(i, j)$ on the lattice plane. When the $i$-th element is a vowel or the syllabic nasal, $L(i, j)$ is calculated successively by the following equations (dynamic programming technique):

$$
\begin{aligned}
L(i, j)= & \max \left[L_{1}(i, j), L_{2}(i, j), L_{3}(i, j), L_{4}(i, j),\right. \\
& \left.L_{5}(i, j), L_{6}(i, j)\right] \\
L_{1}(i, j)= & L^{*}(i-1, j)+S_{0}(i, j) \\
L_{2}(i, j)= & L(i-1, j-1)+S_{0}(i, j) \\
L_{3}(i, j)= & L^{*}(i-1, j-1)+\left[S_{0}(i, j-1)+S_{0}(i, j)\right] / 2 \\
L_{4}(i, j)= & L(i-1, j-2)+\left[S_{0}(i, j-1)+S_{0}(i, j)\right] / 2 \\
L_{5}(i, j)= & L^{*}(i-1, j-2)+\left[S_{0}(i, j-2)+S_{0}(i, j-1)+\right. \\
& \left.S_{0}(i, j)\right] / 3 \\
L_{6}(i, j)= & L(i-1, j-3)+\left[S_{0}(i, j-2)+S_{0}(i, j-1)+\right. \\
& \left.S_{0}(i, j)\right] / 3
\end{aligned}
$$

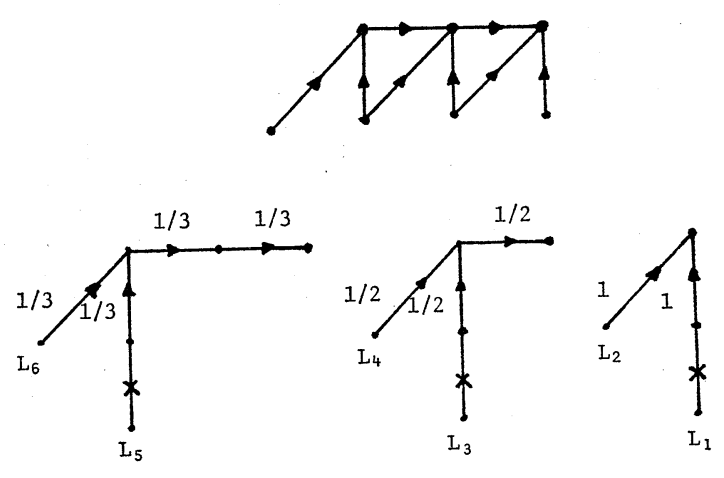

Fig. 6 Possible paths to a grid point and weights of arcs.

If the $i$-th element is marked with the special symbol $(+), L^{*}(i, j)=L(i, j)$; otherwise $L^{*}(i, j)=\max \left[L_{2}\right.$ $\left.(i, j), L_{3}(i, j), L_{4}(i, j), L_{5}(i, j), L_{6}(i, j)\right]$. This selection of $L^{*}(i, j)$ corresponds to the restriction (3) in matching.

The boundary (or initial) conditions are the following:

$$
\begin{aligned}
& L(1, j)=0: j \geqq 4 \\
& L(1,1)=S_{0}(1,1) \\
& L(1,2)=\left[S_{0}(1,1)+S_{0}(1,2)\right] / 2 \\
& L(1,3)=\left[S_{0}(1,1)+S_{0}(1,2)+S_{0}(1,3)\right] / 3
\end{aligned}
$$

When the $i$-th element is a consonant, a silence or an element with the special symbol $(-), L(i, j)$ is calculated by the following equations:

$$
\begin{aligned}
& L(i, j)=\max \left[L_{1}(i, j), L_{2}(i, j), L_{3}(i, j), L_{4}(i, j)\right] \\
& L(1,3)=0
\end{aligned}
$$

Figure 6 illustrates the possible paths to a grid point and the weights of the arcs of the paths (see the notation of the Ref. 10)).

If the numbers of elements in a lexical entry and input string are $i_{0}$ and $j_{0}$, respectively, the likelihood of this word is calculated as $L\left(i_{0}, j_{0}\right) / i_{0}$. The input utterance is recognized as a word which has the highest likelihood out of all words in the dictionary. Figure 7 shows the graphic representation of word matching. This word matching procedure has developed for the word identification procedure of our speech understanding system. ${ }^{11)}$

\section{RECOGNITION OF CONNECTED SPOKEN WORDS}

\subsection{Word Boundary Detection Procedure}

The word boundary of connected spoken words is detected by the procedure as shown in Fig. 8. 

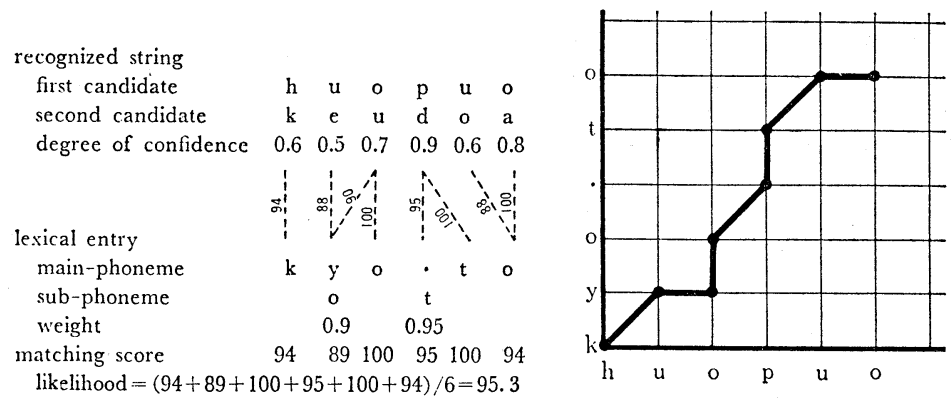

Fig. 7 Graphic representation of an example of word matching and matching score.

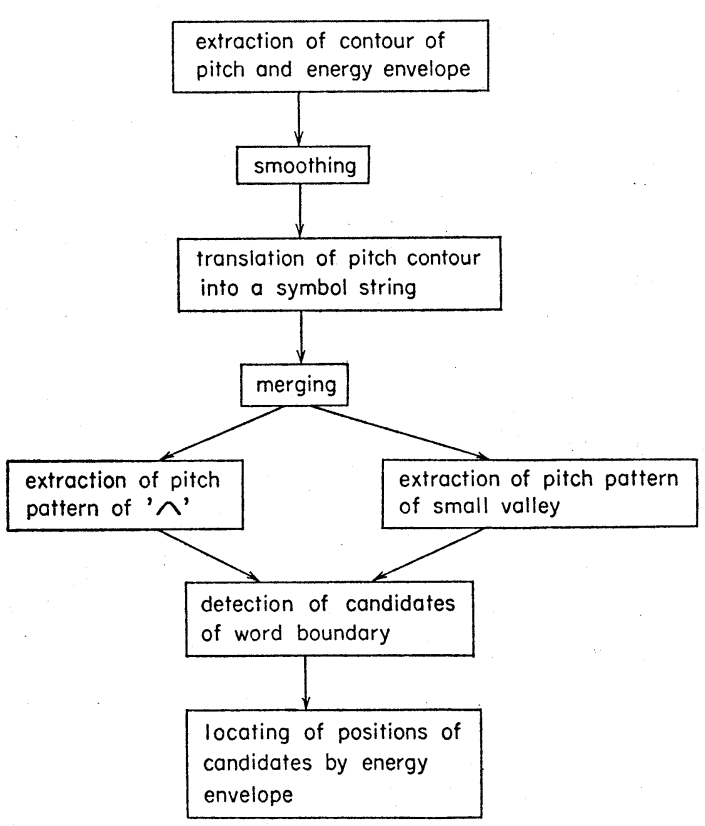

Fig. 8 Word boundary detection procedure.

First, the system decides the preliminary candidates of the word boundary by the use of the pitch contour. Secondly, it decides the final candidates by the use of the valley of the energy envelope. The pitch standard pattern is one that is made by the natural outflow of expiration to utter the word or phrase. The pattern is like the figure of " $\wedge$ ". We think that a word in connected spoken words corresponds to a phrase in continuous speech on pitch contours. Therefore we can detect the word boundary by finding the pattern in the pitch contour. The energy envelope shows a valley at the word boundary, so we can locate the word boundary more accurately. In perceptual experiments, we found that human listeners could detect the number of words in connected spoken digits or city names at the correct rate of about $95 \%$ by using only the information of pitch and energy envelope contours, without using the phonemic information. ${ }^{16)}$

\subsubsection{Detection of candidates by pitch contour}

The pitch extractor which has three channels of low-pass filter (cut-off frequencies are 150, 250, $400 \mathrm{~Hz}$, respectively) and suppressive circuits, receives speech signals and transforms it into a quasi-sine wave whose period corresponds to the pitch period of the speech, if they contained a component of low-frequencies which range from 80 to $400 \mathrm{~Hz}$ in the speech. The pitch period is transformed into a fundamental frequency $\left(F_{0}\right)$ every $10 \mathrm{~ms}$ interval. This output sequence is smoothed by a 5-point median smoothing method. ${ }^{13)}$ To treat a global pitch pattern, the smoothed sequence is sampled at every $30 \mathrm{~ms}$ interval (down sampling). Further, this new sequence, $F 0(1), F 0(2), \ldots$, is transformed into a 5 -value symbol sequence by the slope of pitch contour as follows.

$$
\begin{aligned}
& \text { If } \alpha \leqq F 0(i)-F 0(i-1), \\
& \text { If } 0 \leqq F 0(i)-F 0(i-1)<\alpha, \text { then } F 0(i) \rightarrow+ \\
& \text { If } \beta \leqq F 0(i)-F 0(i-1)<0, \text { then } F 0(i) \rightarrow 0^{-} \\
& \text {If } F 0(i)-F 0(i-1)<\beta, \text { then } F 0(i) \rightarrow- \\
& \text { Otherwise, } \\
& F 0(i) \rightarrow \Lambda
\end{aligned}
$$

That is, if either $F O(i)$ or $F O(i-1)$ is not defined or zero, $F O(i)$ is transformed into a null symbol. This corresponds to unvoiced sounds or silence. For convenience' sake, the symbol ' 0 ' represents either ' $0^{+}$' or ' 0 ''. If the successive symbols are same, they are merged. If there are three or more successive symbols of ' $0^{+}$' (or ' $0^{-}$'), they are replaced by the symbol of ' + ' ( or ' $^{\prime}$ '). The rules of merging process are shown in Fig. 9. The output of this process 


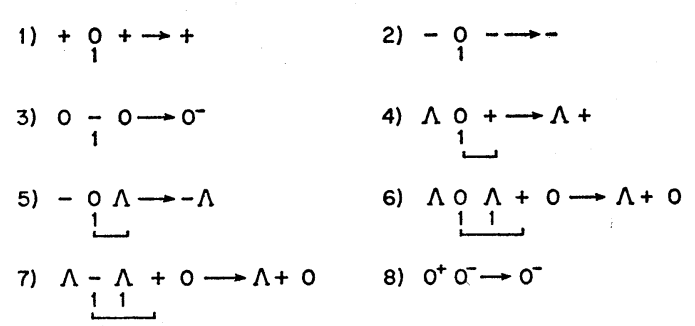

Fig. 9 Merging rules for a symbol sequence. The unit of length is $30 \mathrm{~ms}$.

consists of a sequence of three tuples; symbol, duration and mean value of inclination of pitch contour.

If there exists a valley of pitch pattern, it is considered that there is a boundary, because the pitch contour becomes a dip at almost all the boundaries. However this dip does not sometimes appear. Therefore, we adopted a strategy which found the pitch pattern of ' $A$ ', not ' $\checkmark$ '. Figure 10 shows the rewriting rules. ' $W$ ' corresponds to the pitch pattern of a word. Both ends of a word pitch pattern ' $W$ ' are regarded as preliminary candidates of word boundary. However, these rules overlook the small dip of pitch pattern. Thus, the system also adopts the another rule; a small dip between ' $0^{-}$' and ' $0^{+}$' is also regarded as a candidate of a word boundary.

6.1.2 Location of candidates by energy envelope The pitch contour usually sifts to a right direction (delay) in comparison with the intention of a speaker, or linguistic acoustic phenomenon. But fortunatelly, the energy envelope corresponds to that phenomenon. Thus, the energy envelope is used to locate the word boundary more accurately, since the envelope usually shows a valley at the word boundary. But it shows delicate changes in the unvoiced consonant when a word begins at an unvoiced consonant, so the system considers the case. The search range of valley is from the point preceding $150 \mathrm{~ms}$ of a preliminary candidate to the point following $90 \mathrm{~ms}$. If there is a valley, the point is regarded as a final candidate of word boundary.

\subsection{Recognition Procedure}

\subsubsection{Extraction of word sequence}

For convenience' sake, we restrict to simplify the algorithm that the number of words in connected words is no more than 3 . Therefore the system assumes that an input utterance contains one, two,
1) $\Lambda+0 \rightarrow \Lambda w$
4-10
3) $\wedge 0 \rightarrow \wedge w$
4-10
5) $\Lambda \circ \wedge 0^{*} \Lambda \longrightarrow \wedge w \wedge$
$5-10$

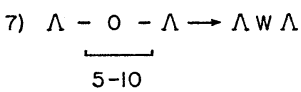
8) $\Lambda \circ \wedge \rightarrow \Lambda w \Lambda$
$\underset{5-10}{\sim}$
9) $w \rightarrow w$
5-10
II) $w+0 \rightarrow w w$
4-10
13) $w \underbrace{\circ \Lambda 0^{*}}_{5-10} \Lambda \rightarrow w w \Lambda$
10) $w+-\longrightarrow w w$
$\underset{4-10}{\longrightarrow}$
12) $\underset{4-10}{\mathrm{w}} \underset{4}{\mathrm{a}} \underset{\mathrm{C}}{\mathrm{O}} \longrightarrow \mathrm{w} w$
15) $w \circ \Lambda \rightarrow W W$ 5-10
14) $w \underset{5-10}{\circ{ }^{*} \Lambda \rightarrow w W} \Lambda$
16) $\underset{1}{\mathrm{w}} \underset{\mathrm{1}}{0} \Lambda \rightarrow w \Lambda$

Fig. 10 Rewriting rules which find the pitch patterns of words.

The unit of length is $30 \mathrm{~ms}$. The symbol '*' denotes that there should be the difference of pitch frequency more than $+10 \mathrm{~Hz}$ between the end point of preceding voiced sound and the front point of following voiced sound. The condition of duration assumes that the vocabulary is ten digits.

or three words. Let $B_{s}$ and $B_{f}$ be the beginning point and ending point of 'speech' of an input, respectively. Now, let $n$ be the detected number of candidates of word boundary and also $B_{1}, \ldots, B_{n}$ be their points in connected speech, respectively. The input utterance is divided into several parts according to $n$ as follows, each of which may corresponds to a word.

(i) $n=0$

The input is regarded as consisting of one word, that is,

(ii) $n=1$

$$
B_{s}-B_{f}
$$

The input is regarded as consisting of one or two words, that is,

$$
B_{s}-B_{f}
$$


(iii) $n=2$

$$
\text { or } B_{s}-B_{1}, \quad B_{1}-B_{f}
$$

The input is regarded as consisting of two or three words, that is,

$$
\begin{array}{lll} 
& B_{s}-B_{1}, & B_{1}-B_{f} \\
\text { or } & B_{s}-B_{2}, & B_{2}-B_{f} \\
\text { or } & B_{s}-B_{1}, & B_{1}-B_{2}, \\
\end{array}
$$

(iv) $n=3$

The input is regarded as consisting of two or three words, that is,

(v) $n=4$

$$
\begin{aligned}
& B_{s}-B_{1}, \quad B_{1}-B_{f} \\
& \text { or } B_{s}-B_{2}, \quad B_{2}-B_{f} \\
& \text { or } B_{s}-B_{3}, \quad B_{3}-B_{f} \\
& \text { or } B_{s}-B_{1}, \quad B_{1}-B_{2}, \quad B_{2}-B_{f} \\
& \text { or } B_{s}-B_{1}, \quad B_{1}-B_{3}, \quad B_{3}-B_{f} \\
& \text { or } B_{s}-B_{2}, B_{2}-B_{3}, \quad B_{3}-B_{f}
\end{aligned}
$$

The input is regarded as consisting of three words, that is,

$$
\begin{array}{llll} 
& B_{s}-B_{1}, & B_{1}-B_{3}, & B_{3}-B_{f} \\
\text { or } & B_{s}-B_{2}, & B_{2}-B_{3}, & B_{3}-B_{f} \\
\text { or } & B_{s}-B_{2}, & B_{2}-B_{4}, & B_{4}-B_{f}
\end{array}
$$

\subsubsection{Modification of classified phoneme string}

The portion of a word is extracted by the above procedure, then the classified phoneme string which corresponds to that portion is extracted by the following procedure.

(i) If the shortest difference between the extracted word boundary and classified phoneme boundaries is shorter than $30 \mathrm{~ms}$, the phoneme boundary is regarded as a word boundary.

(ii) If the shortest difference is longer than $30 \mathrm{~ms}$, a classified phoneme is divided into two segments at the point of extracted word boundary.

Thus, the system can extract a classified phoneme string corresponding to one word. However, the word boundary detection procedure as mentioned in section 6.1 often yields a shift of one phoneme caused by the symbol of ' $\Lambda$ ', that is, unvoiced sounds. Therefore the extracted phoneme string is modified as follows. Let $F_{1} F_{2} \ldots F_{s} \ldots F_{t} \ldots F_{n}, S_{1} S_{2} \ldots S_{s}$ $\ldots S_{t} \ldots S_{n}$ and $P_{1} P_{2} \ldots P_{s} \ldots P_{t} \ldots P_{n}$ be the first candidate sequence of phonemes, the second candidate sequence and the reliability sequence of the first candidate, respectively. When the phoneme string $s$ to $t$ corresponds to a word,

(i) if $F_{s}$ is a vowel and $F_{s-1}$ is an unvoiced consonant,

$$
S_{s} \leftarrow F_{s-1}, \quad P_{s} \leftarrow 0
$$

(ii) if $F_{s}$ is an unvoiced consonant,

$$
S_{s} \leftarrow F_{s+1}, \quad P_{s} \leftarrow 0
$$

(iii) if $F_{t}$ is a vowel and $F_{t+1}$ is an unvoiced consonant,

$$
S_{t} \leftarrow F_{t+1}, \quad P_{t} \leftarrow 0
$$

(iv) if $F_{t}$ is an unvoiced consonant,

$$
S_{t} \leftarrow F_{t-1}, \quad P_{t} \leftarrow 0
$$

This new modified phoneme string is transformed to a word by the word matching procedure as described in section 5 .

\subsubsection{Evaluation of word lattice}

By the method mentioned above, several word sequences are generated. The system should select the best word sequence as the recognition result. We adopted the following criterion for the selection. To take preference the longer word sequence than the shorter word sequence, the system adds one point to the average score for two connected words, and two points for three connected words. From all of such new average scores, the system selects the word sequence with the highest average score and it is regarded as the input utterance.

\section{SYSTEM EVALUATION}

We applied our system to the speech recognition of 100 city names in Japan and connected digits. The reference spectral patterns of phonemes and similarity matrix (Table 1) were calculated from VCV contexts which were included in 2450 words spoken by 10 male adults $(245 \mathrm{VCV}$ syllable $\times 10)$. The local bit patterns for pre-matching were obtained from Japanese $100 \mathrm{CV}$ syllables spoken in isolation. The experiments described here did not use any learning procedure of speaker differences. The classification rates of vowels, unvoiced consonants and voiced consonants were about $78 \%, 70 \%$ and $30 \%$, respectively.

\subsection{Experiment of Pre-Matching}

In order to investigate the effectiveness of prematching, we made experiments to recognize the spoken words of 100 Japanese city names, or 10 digits. 
Table 3 Recognition results of isolated spoken words.

(a) 100 Japanese city names $(100 \times 5$ samples $)$.

\begin{tabular}{ccccccrrr}
\hline Speaker & $\begin{array}{c}\mathrm{a} \\
(\%)\end{array}$ & $\begin{array}{c}\mathrm{b} \\
(\%)\end{array}$ & $\begin{array}{c}\mathrm{c} \\
(\%)\end{array}$ & $\begin{array}{c}\mathrm{d} \\
(\%)\end{array}$ & $\begin{array}{r}\mathrm{e} \\
(\%)\end{array}$ & $\mathrm{f}$ & $\mathrm{g}$ \\
\hline ST & 55.0 & 70.0 & 71.0 & 80.0 & 86.0 & 9.23 & 8 \\
FK & 63.0 & 71.0 & 73.0 & 76.0 & 83.0 & 10.20 & 10 & \\
HR & 88.0 & 86.0 & 90.0 & 92.0 & 94.0 & 9.96 & 4 & 6 \\
MK & 70.0 & 76.0 & 78.0 & 86.0 & 92.0 & 8.82 & 5 \\
MG & 76.0 & 80.0 & 83.0 & 84.0 & 90.0 & 10.46 & 9.73 & 6.6 \\
Average & 70.4 & 76.6 & 79.0 & 83.6 & 89.0 & 9.0 \\
\hline
\end{tabular}

(b) 10 Japanese digits $(10 \times 2 \times 5$ samples $)$.

\begin{tabular}{cccccccccc}
\hline Speaker & $\begin{array}{c}\mathrm{a} \\
(\%)\end{array}$ & $\begin{array}{c}\mathrm{b} \\
(\%)\end{array}$ & $\begin{array}{c}\mathrm{c} \\
(\%)\end{array}$ & $\begin{array}{c}\mathrm{d} \\
(\%)\end{array}$ & $\begin{array}{c}\mathrm{d}^{*} \\
(\%)\end{array}$ & $\mathrm{f}$ & $\mathrm{g}$ & $\mathrm{g}^{*}$ \\
\hline NG & 100 & 100 & 100 & 100 & 100 & 2.8 & 0 & 0 \\
UK & 95 & 85 & 95 & 100 & 100 & 2.8 & 0 & 0 \\
AR & 90 & 95 & 90 & 95 & 100 & 2.7 & 1 & 0 \\
NK & 85 & 90 & 85 & 85 & 90 & 3.2 & 1 & 0 \\
TR & 85 & 85 & 85 & 95 & 100 & 2.9 & 1 & 0 \\
Average & 91 & 91 & 91 & 95 & 98 & 2.9 & 0.6 & 0 \\
\hline
\end{tabular}

Table 3 shows the results.

The column (a) shows the correct rate of recognition in the case without pre-matching, that is, with using only word matching.

The column (b) shows the correct rate in the case without word matching, that is, with using only pre-matching.

The column (c) shows the correct rate in the case with the pre-selection by pre-matching and with word matching.

The column (d) shows the correct rate in the case with the pre-selection and with the final decision by adding the pre-matching score to word matching score.

The column (e) shows the correct rate within the top 2 choices in the case (d).

The column (f) shows the average number of preselected words.

The column ( $\mathrm{g}$ ) shows the rejected number of input words by the pre-selection, that is, these input words were misrecognized by the stage of prematching.

In spoken digits, since ' $u$ ' of 'roku (six)' is often devocalized, the local bit pattern at the tail position is different from the standard bit pattern of the isolated syllable 'ku'. Therefore we did not use the local bit pattern of the syllable ' $k u$ ', but used the pattern of 'roku'. The results of this case are shown in the columns $\left(\mathrm{d}^{*}\right)$ and $\left(\mathrm{g}^{*}\right)$.

From these results, we can conclude that the preselection by pre-matching reduced the candidate words from 100 words to about 10 words. In other words, the recognition time was improved a factor of 10 , since the times of phoneme recognition and prematching can be ignored in comparison with word matching.

And also, the correct rate was improved from about $70 \%(91 \%)$ to about $84 \%(98 \%)$ for 100 city names (10 digits) by taking the pre-matching into consideration. However, a few correct words were rejected by the pre-selection.

\subsection{Experiment of Recognition of Connected Spoken Digits}

Next, we experimented on the recognition of connected digits spoken by four male adults. Each speaker uttered 10 isolated-digits (one time per digit), 100 two-connected-digits (all pairs) and 100 three-connected-digits (selection from 1000 strings at random), respectively. The number of digits in an input utterance was restricted up to 3 . The results are tabulated in Table 4.

The column (a) shows the number detected as candidates of word boundary. The total number in 
Table 4 Recognition results of connected digits.

\begin{tabular}{|c|c|c|c|c|c|c|c|c|}
\hline \multirow{2}{*}{ Speaker } & \multicolumn{2}{|c|}{ Isolated } & \multicolumn{3}{|c|}{2 connected } & \multicolumn{3}{|c|}{3 connected } \\
\hline & $\mathrm{a}$ & $c(\%)$ & $\mathrm{a}$ & b & $c(\%)$ & $\mathrm{a}$ & b & $\mathrm{c}(\%)$ \\
\hline NK & 3 & 100 & 168 & $92(95)$ & 88 & 265 & 177 (181) & 76 \\
\hline IN & 4 & 90 & 167 & $97(98)$ & 88 & 276 & 184 (191) & 79 \\
\hline MS & 2 & 100 & 137 & $88(91)$ & 70 & 240 & $170(177)$ & 63 \\
\hline MZ & 2 & 90 & 139 & $90(95)$ & 87 & 271 & 190 (194) & 84 \\
\hline Average & 2.8 & 95 & 153 & $92(95)$ & 83 & 263 & $180(186)$ & 76 \\
\hline
\end{tabular}

real utterances is 0 for isolated digits, 100 for twoconnected digits and 200 for three-connected digits, respectively. The column (b) shows the number detected correctly. The number in parentheses contains the sift of only one phoneme in a classified phoneme string. Such a shift may be correctly processed by the modification of a classified phoneme string described in section 6.2(b). The column (c) shows the recognition rate.

In the case of recognition of isolated digits, the pre-selection by pre-matching reduced the candidate words from 10 words to about 3 words (refer to Table 3(b)). However, the contour of energy envelope was not used for pre-matching in the case of two and three connected digits. Furthermore, the local patterns were not also used in the case of three-connected digits. Because it is difficult to make patterns of the pre-matching for connected spoken words.

The number detected as candidates of word boundary was $1.2 \sim 1.7$ times of the real number and the boundary was detected correctly at the rate of $85 \sim 95 \%$. In the case of connected digits consisting of only voiced sounds, the rate ranged from 90 to $99 \%$. From this result, we found it was necessary to take unvoiced sounds into consideration.

The performance of word boundary detection is not sufficient for our recognition strategy, because our strategy is based on the result of word boundary detection. If this detection procedure is used in aid of a general connected word recognition system, it will be useful to reduce the amount of computation or to confirm the word boundary results such as the use of phrase boundary detection procedure in continuous speech. ${ }^{18)}$

\section{A SPOKEN WORD RECOGNIZER ${ }^{14)}$}

We constructed a special equipment of speech recognition on a micro-computer which was based on our speech recognition system developed on the mini-computer. This equipment is an isolated spoken word recognizer on the basis of phoneme recognition and as a priori knowledge, has the reference pattern and duration for each phoneme, word dictionary, phoneme similarity, phonemic system, phonological rule and so on. And also it has a prematching procedure for reducing the recognition time and a learning function of speaker differences for unspecified speakers.

Table 5 summarizes the differences between our speech recognition system and the recognizer. Since the power of micro-computer is smaller than that of mini-computer, some functions are eliminated and some algorithms are simplified. The eliminated functions are the classification of unvoiced consonants, the normalization of coarticulation and the uses of number of phonemes and phonemic pattern for prematching. In this section, we describe about the recognizer on the different points.

\subsection{Recognition Process}

Figure 11 shows the recognition process. The labeling procedure (phoneme classification) is performed to every frame. The three consecutive label sequence is merged into one label for reducing the processing time at the word matching stage. After this processing, if one or two labels remain at the tail part of a label sequence, they are abandoned. However, these spectral features are preserved as a local bit pattern for pre-matching. Thus, the output sequence is composed by about 10 30 labels for $300 \sim 900 \mathrm{~ms}$ of duration of a spoken word. The label of each segment consists of 3 tuples; the first candidate phoneme, the second candidate phoneme and the reliability of the first candidate.

If a frame of a silence part is detected, a local bit pattern and an energy envelope pattern are generated and then the pre-matching and word matching 
S. NAKAGAWA and T. SAKAI: A SPOKEN WORD RECOGNITION SYSTEM AND RECOGNIZER

Table 5 Comparison between recognition system and recognizer.

\begin{tabular}{|c|c|c|}
\hline & Recognition system & Recognizer \\
\hline CPU & MELCOM-70 & $6800 \mathrm{H}$ \\
\hline (Main memory) & $32 \mathrm{~kW}$ (16 bits/word) & ROM 8 kB, RAM 8 kB \\
\hline (Auxiliary memory) & $2.5 \mathrm{MW}$ (disk file) & ROM $32 \mathrm{kB}$, RAM $16 \mathrm{kB}$ \\
\hline (Cycle time) & $0.8 \mu \mathrm{s}$ & $2.0 \mu \mathrm{s}$ \\
\hline Acoustic processor & $\begin{array}{l}20 \text { channel } 1 / 4 \text { octave } \\
\text { filter bank } \\
\text { pitch extractor (Halsey type) }\end{array}$ & $\begin{array}{l}20 \text { channel } 1 / 4 \text { octave } \\
\text { filter bank }\end{array}$ \\
\hline Segmentation & phoneme by phoneme & $\begin{array}{l}\text { unvoiced/vowel/voiced consonant } \\
\text { frame by frame }\end{array}$ \\
\hline $\begin{array}{l}\text { Phoneme } \\
\text { classification }\end{array}$ & $\begin{array}{l}\text { unvoiced consonant: } \\
\text { decision tree } \\
\text { vowel: } \\
\text { linear discriminant function } \\
\text { voiced consonant: } \\
\text { Euclidean distance }\end{array}$ & $\begin{array}{l}\text { unvoiced consonant: } \\
\text { decision tree (plosive/fricative) } \\
\text { vowel: Euclidean distance } \\
\text { voiced consonant: } \\
\text { Euclidean distance }\end{array}$ \\
\hline Word dictionary & $\begin{array}{l}\text { main-phoneme } \\
\text { sub-phoneme } \& \text { weight }\end{array}$ & $\begin{array}{l}\text { main-phoneme } \\
\text { sub-phoneme \& weight, duration }\end{array}$ \\
\hline Pre-matching & $\begin{array}{l}\text { local spectral pattern } \\
\text { energy envelope pattern } \\
\text { number of phonemes } \\
\text { phonemic pattern }\end{array}$ & $\begin{array}{l}\text { local spectral pattern } \\
\text { energy envelope pattern }\end{array}$ \\
\hline Word matching & $\begin{array}{l}\text { asymmetric DP matching } \\
\text { normalization of coarticulation }\end{array}$ & symmetric DP matching \\
\hline $\begin{array}{l}\text { Learning of } \\
\text { speaker differences }\end{array}$ & $\begin{array}{l}\text { isolated vowel } \\
\text { supervised } \\
\text { non-supervised } \\
\text { estimation of voiced } \\
\quad \text { consonant's spectrum } \\
\text { modification of phoneme similarity }\end{array}$ & $\begin{array}{l}\text { isolated vowel } \\
\text { supervised } \\
\text { non-supervised }\end{array}$ \\
\hline Speech data & abandom by next input frame & preserve until next input word \\
\hline Utterance & isolated/connected & isolated \\
\hline Portability & no & yes \\
\hline
\end{tabular}

procedures are invoked. If the duration of the silence part is less than $300 \mathrm{~ms}$ ( 30 frames), the silence is regarded as a choked sound or a closure of plousive consonant. Therefore the above procedures are interrupted. If it is over $300 \mathrm{~ms}$, the silence is regarded as a nonspeech part, that is, the end of an input utterance, and then the recognition result is displayed.

At the pre-matching stage, the recognizer uses the binary patterns of spectra in the head and tail posi- tion of a spoken word ( 60 bits) and the binary pattern of contour of energy envelope as described in section 4. Although the recognition system divides the length of a word into 14 intervals linearly, the recognizer into 12 intervals ( 24 bits $=3$ bytes). Thus Hamming distance by the pre-matching ranges from 0 to $84(60+24)$.

The final decision of recognition is performed by the total score of pre-matching score (maximum point $=84 \times 3$ ) and word matching score (maximum 


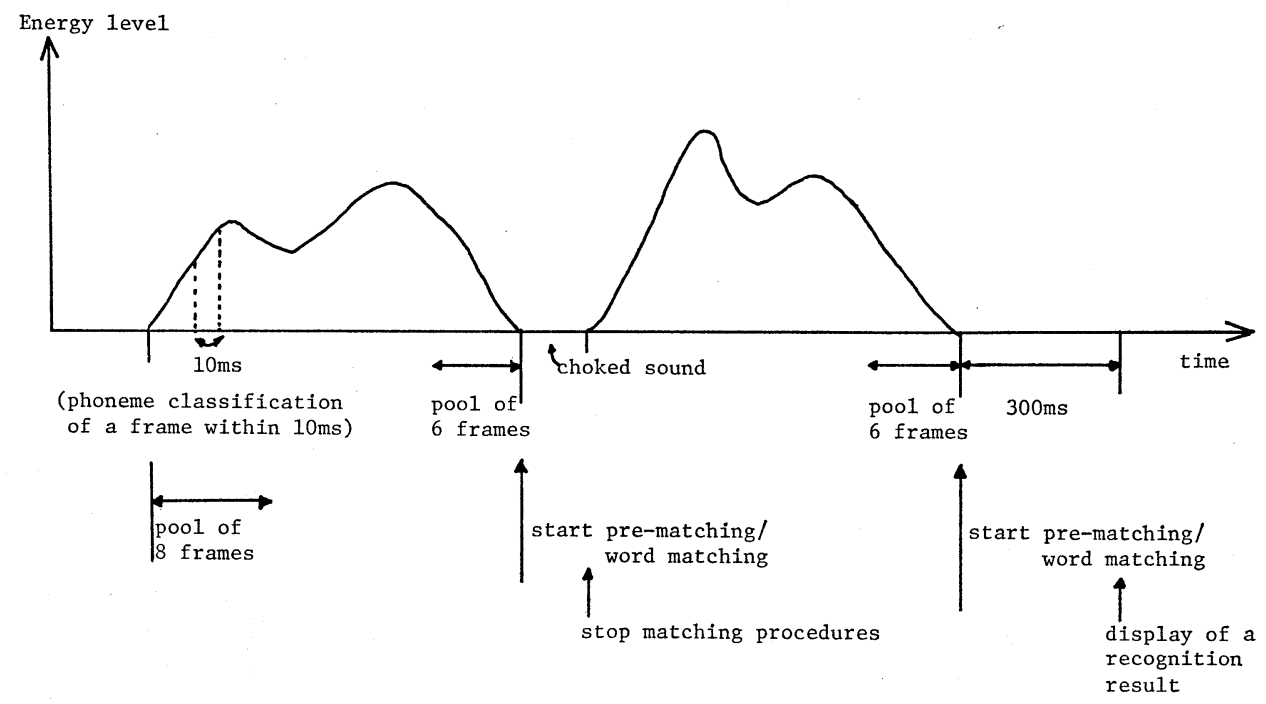

Fig. 11 Recognition process of recognizer.

point $=100 \times 10$ ). If the total score is less than 1000 points, the decision of an utterance is rejected.

\subsection{Word Matching}

An element by which a word in the word dictionary is described is either a main-phoneme or a subphoneme plus a weighting factor as shown in section 5.3. In the recognition system, a lexical word is represented by a sequence of elements. The sequence usually corresponds to a pronouncing manner as shown in Table 2. In the recognizer, however, the sequence has explicitly the knowledge of phonemic duration. Figure 12 shows an lexical example. The number of elements corresponds to the duration of spoken word, that is, the number of classified labels. Therefore, the word matching algorithm by dynamic programming (DP matching) is changed into the following (refer to section 5.3).

$$
\begin{aligned}
& L(i, j)=S_{0}(i, j) \\
& +\max \left\{\begin{array}{l}
L(i-2, j-1)+2 \cdot S_{0}(i-1, j) \\
L(i-1, j-1)+S_{0}(i, j) \\
L(i-1, j-2)+2 \cdot S_{0}(i, j-1)
\end{array}\right\} \\
& L(1,1)=S_{0}(1,1) \\
& L(1,2)=L(1,1)+S_{0}(1,2) \\
& L(2,1)=L(1,1)+S_{0}(2,1) \\
& {[n \cdot j / m+0.5]-2<i<[n \cdot j / m+0.5]+2}
\end{aligned}
$$

The likelihood of this word is calculated as $L\left(i_{0}, j_{0}\right) /$

$\begin{array}{lcc}\text { main-phoneme } & \text { i } \cdot \text { c } & \text { i } \\ \text { sub-phoneme } & \text { c } & \text { c } \\ \text { weight } & 1.0 & 1.0\end{array}$

(a) recognition system

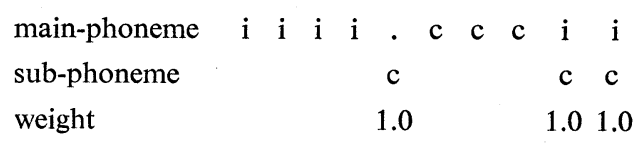

(b) recognizer

Fig. 12 Lexical entry of 'ichi (one).'

$\left(i_{0}+j_{0}-1\right)$. This algorithm is the same one proposed by Sakoe and Chiba. ${ }^{15)}$

\subsection{Evaluation of Recognizer}

In order to evaluate our speech recognizer, we made an experiment to recognize the spoken words of 32 Japanese city names. The vocabulary was selected at random from 100 city names described in section 7.1. Ten adult speakers, five males and five females, participated in the experiment and each of them uttered the 32 word vocabulary ten times. The reference spectral patterns of phonemes were also prepared for female speakers, but the phoneme similarity matrix, the local bit patterns and the energy patterns for pre-matching were shared for both male and female speakers. 


\section{S. NAKAGAWA and T. SAKAI: A SPOKEN WORD RECOGNITION SYSTEM AND RECOGNIZER}

Table 6 Recognition results of 32 city names by recognizer.

\begin{tabular}{ccccc}
\hline \multicolumn{2}{c}{ Male } & & \multicolumn{2}{c}{ Female } \\
\cline { 1 - 2 } \cline { 5 - 5 } Speaker & $\begin{array}{c}\text { Recognition } \\
\text { rate }(\%)\end{array}$ & & Speaker & $\begin{array}{c}\text { Recognition } \\
\text { rate }(\%)\end{array}$ \\
\hline MY & 96.6 & & FO & 93.8 \\
MT & 97.5 & & FM & 93.1 \\
MO & 96.3 & & FK & 93.1 \\
MN & 93.4 & & FI & 95.0 \\
MK & 87.5 & & FH & 91.6 \\
\cline { 1 - 1 } Average & 94.3 & & Average & 93.3
\end{tabular}

Table 6 shows the recognition results. The overall accuracies were $94.3 \%$ for male speakers and $93.3 \%$ for female speakers. Some utterances were rejected at the stage of pre-matching. If we use the multiple bit patterns for pre-matching or the learning is performed for the speaker difference, these rejection may be avoided. Moreover, the multiple reference patterns for each phoneme may be necessary to recognize utterances spoken by unspecified speakers.

The response time was about one second after the utterance. Figure 13 shows the photograph of this recognition equipment. This consists of recognizer in itself, display, touch keyboard and microphone. The level of input speech is monitored by the lamp. The keyboard is used to make a dictionary and to control the recognizer.

\section{CONCLUSION}

We proposed a pre-matching method to eliminate the most unlikely group of candidates using the measurements of both local and global features of a spoken word from the vocabulary list. This operation also made increase the correct rate of recognition. On experiment of recognition of isolated 100 Japanese city names or digits, the system recognized them in real time with the correct rate of $84 \%$ or $98 \%$ for unspecific speakers, respectively.

Further, we proposed a new recognition method of connected spoken words, which was based on the detection of word boundary. It was performed by using the contours of pitch pattern and energy envelope. The system could detect the word boundaries in two and three connected digits at the rate of more than $90 \%$. However, the recognition rate

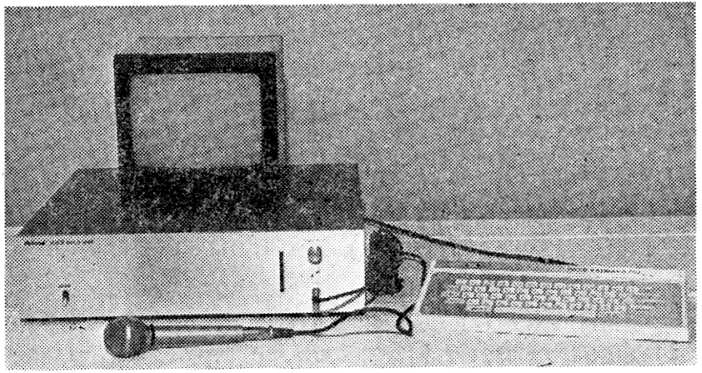

Fig. 13 A spoken word recognizer. Upper: display unit, lower: speech recognizer (filter bank, micro-computer), right: touch keyboard.

decreased to about $83 \%$ for two connected digits.

Finally, we constructed a spoken word recognition equipment based on the recognition system. This equipment consisted of the 20 channel $1 / 4$ octave filter bank and a micro-computer, and could recognize 32 city names correctly at the rate of $93.4 \sim$ $94.4 \%$ for unspecific male and female speakers. This recognizer is not useful for only industrial fields but also the learning of Japanese, because we can know the various kinds of differences between an input utterance and the standard utterance by displaying the results of recognition process.

Of course, the recognition system and the recognizer have the more performance for a limited speaker by using the learning procedure of speaker differences, ${ }^{1,2,17)}$ not described in this paper.

\section{ACKNOWLEDGEMENT}

The authors wish to thank Mr. M. Utsumi for his cooperation of pre-matching, Mr. H. Nakase for the development of connected word recognition method and Mr. E. Tsuboka et al. (members of Electronics Research Laboratory, Matsushita Electric Industrial Co., Ltd.) for the development of the recognizer. This research was partly supported by the Grant for Scientific Researchers from the Ministry of Education of Japan.

\section{REFERENCES}

1) S. Nakagawa and T. Sakai, "A real time spoken word recognition system with various learning capabilities of the speaker differences," IECEJ Trans. 61-D, 395-403 (1978) (in Japanese).

2) S. Nakagawa and T. Sakai, "A real time spoken word recognition system in a large vocabulary with 
learning capability of speaker differences," Proc. 4th IJCPR, 985-989 (1978).

3) D. R. Reddy, "Speech recognition by machine: a review," Proceedings of the IEEE, 64, 501-531 (1976).

4) S. Chiba, M. Watari, and T. Watanabe, "A speakerindependent word recognition system," Proc. 4th IJCPR, 995-999 (1978).

5) L. R. Rabiner and M. R. Sambur, "Some preliminary experiments in the recognition of connected digits," IEEE Trans. ASSP-24, 170-182 (1976).

6) M. F. Medress, T. E. Skinner, D. R. Kloker, T. C. Diller, and W. A. Lea, "A system for the recognition of spoken connected word sequence," Conference record of 1977-ICASSP, 468-473 (1977).

7) H. Sakoe, "Two-level DP-matching-a dynamic programming-based pattern matching algorithm for connected word recognition," IEEE Trans. ASSP-27, 588-595 (1979).

8) R. Nakatsu and M. Kohda, "Speech recognition of connected words," Proc. 4th IJCPR, 1009-1011 (1978).

9) S. Nakagawa and T. Sakai, "Feature analyses of Japanese phonetic spectra and considerations on speech recognition and speaker identification," J. Acoust. Soc. Jpn. 35, 111-117 (1979) (in Japanese).

10) C. Myers, L. R. Rabiner, and A. E. Rosenberg, "Performance tradeoffs in dynamic time warping algorithms for isolated word recognition," IEEE Trans. ASSP-28, 623-635 (1980).

11) T. Sakai and S. Nakagawa, "A classification method of spoken words in continuous speech for many speakers," J. Inform. Proc. Soc. Jpn. 17, 650-658 (1976) (in Japanese), or Inform. Proc. Jpn. 17, 613 (1977).

12) M. Ichino and K. Hiramatsu, "Feature effectiveness criteria of statistical pattern classifier," IECEJ Trans. 53-C, 748-755 (1970) (in Japanese).

13) L. R. Rabiner, M. R. Sambur, and C. E. Schmidt, "Applications of a nonlinear smoothing algorithm to speech processing," IEEE Trans. ASSP-23, 552-557 (1975).

14) S. Nakagawa, T. Sakai, and E. Tsuboka, "A spoken word recognizer for unlimited speakers on the basis of phoneme recognition," Tech. Group Speech, Acoust. Soc. Jpn. Paper S80-62 (1980) (in Japanese).

15) H. Sakoe and S. Chiba, "Dynamic programming algorithm optimization for spoken word recognition," IEEE Trans. ASSP-26, 43-49 (1978).

16) S. Nakagawa and T. Sakai, "A recognition system of connected spoken words based on word boundary detection," Stud. Phonologica XIII, 23-38 (1979).

17) T. Sakai, S. Nakagawa, and S. Hayashi, "Spoken words recognition in a limited vocabulary by preliminary learning of the speaker differences," IECEJ, Tech. Report, EA75-61 (1976) (in Japanese).

18) T. Ukita, S. Nakagawa, and T. Sakai, "A use of pitch contour in recognizing spoken Japanese arithmetic expressions," IECEJ Trans. 63-D, 954-961 (1980) (in Japanese). 